\title{
USING A VIDEO CAMERA-BASED METHOD TO GATHER REAL WORLD HIGH BEAM USAGE DATA
}

\author{
Stephanie A. Whetsel Borzendowski, \\ Ashley A. Stafford Sewall, \& Richard A. Tyrrell \\ Clemson University \\ Clemson, SC, USA \\ stephanie.whetsel@gmail.com
}

\begin{abstract}
Summary: The majority of traffic fatalities involving pedestrians occur at night and this is largely attributed to low illumination conditions. Yet, drivers tend to underuse their high beams despite the visibility benefits afforded to them. In the present study we report high beam usage rates during an open-road drive using a video camera-based method. Measurements of low and high beam headlamp illuminance were also taken for all vehicles used in this study. The results indicate that drivers, on average, used their high beams $48 \%$ of the time possible. Furthermore, there was a moderately negative relationship between low beam output and high beam use indicating that drivers whose low beams produced less illumination tended to use their high beams more often. Future research should empirically investigate this relationship to lend further insight into the mechanism by which beam output influences beam usage. Research that improves our understanding of drivers' knowledge and use of high beams is likely to be important as headlighting technologies continue to advance.
\end{abstract}

\section{INTRODUCTION}

In 2012 in the United States, over 4,700 pedestrians were killed in traffic collisions and almost $70 \%$ of these fatalities occurred at night. Pedestrians' increased risk of involvement in traffic collisions at night is due in large part to the low illumination conditions associated with night driving. Indeed, analyses of the U.S. Fatality Analysis Reporting System (FARS) crash database revealed that as ambient illumination decreases, pedestrian fatalities increase even when other crash factors such as alcohol consumption and driver fatigue are held constant (Owens \& Sivak, 1996; Sullivan \& Flannagan, 2002). Compounding this problem is the fact that many drivers overdrive their headlamps at night (i.e., driving at unsafe speeds given reduced illumination), since low beam headlamps provide insufficient illumination to ensure that drivers will be able to see and respond to hazards in time to avoid collisions (Sullivan \& Flannagan, 2001). Leibowitz, Owens, and Tyrrell (1998) reported that when drivers relied on low beam headlamps while driving $25 \mathrm{mph}$, the stopping distance required to avoid a collision with a pedestrian wearing dark clothing was 1-3x greater than the visibility distance of that pedestrian. Since recognizing pedestrians under low illumination conditions can be particularly difficult for drivers, a reliance on low beam headlamps can significantly increase a pedestrian's risk of fatality at night.

Substantial research (e.g., Allen, Hazlett, Tacker, \& Graham, 1969; Shinar, 1984) has demonstrated that pedestrians' conspicuity to drivers at night can be enhanced by the use of retroreflective material. Recent research (e.g., Balk, Tyrrell, Brooks, \& Carpenter, 2008; Wood, Tyrrell, \& Carberry, 2005) has focused on the conspicuity benefit of configurations that highlight biological motion (biomotion) (i.e., retroreflective markings on the wrists, elbows, shoulders, 
waist, knees, and ankles). Findings from these studies indicate that recognition distances of biomotion-clad pedestrians are significantly and substantially longer than those obtained with other retroreflective configurations (e.g., vest).

While the addition of retroreflective material to pedestrians' clothing provides an effective and potentially simple means by which to improve pedestrian conspicuity at night, the usefulness of retroreflective material is dependent on illumination from headlamps. High beams are designed to project greater illumination on the roadway, including onto the roadway that is farther ahead of the vehicle (Rumar, 2000). High beam headlamps afford substantial visibility benefits due to the aim of the beams as well as increased illumination projected on the roadway, providing critical safety benefits to drivers (Helmers \& Rumar, 1975).

Previous research (e.g., Olson \& Sivak, 1983; Roper \& Howard, 1937; Wood, Tyrrell, \& Carberry, 2005) has documented the limitations of low beam headlamps and the increased visibility afforded by high beam headlamps. Roper and Howard (1937) found that decreased candlepower combined with low contrast clothing worn by pedestrians drastically decreased a drivers' ability to detect pedestrians. These results suggest that both pedestrians' clothing and headlamp illumination are factors relevant to conspicuity. More recently, Wood, Tyrrell, and Carberry (2005) reported that the mean recognition distance of a pedestrian increased by a factor of 1.6x on average when drivers used high beams. High beams were particularly useful for the clothing conditions that did not include retroreflective material as response distances increased, on average, by a factor of $2.7 x$.

Despite the significant visibility advantages provided by high beams, they are often under-used by drivers. Hare and Hemion (1968), the first to measure real world beam usage, reported that drivers used high beams less than $25 \%$ of the time that high beams could have been safely used (i.e. improved forward visibility without creating a source of glare for oncoming drivers). More recent studies have confirmed the finding that drivers underuse their high beams. Sullivan, Adachi, Mefford, and Flannagan (2004) also observed beam usage (on two lane, rural roadways with no fixed illumination present) and judged only "clear vehicles" (i.e., no opposing, leading, or following vehicles present). For the observed vehicles high beams were only used half of the time, despite ideal conditions for high beam usage. Both Mefford, Flannagan, and Bogard (2006) and Buonarosa, Sayer, and Flannagan (2008) measured beam usage by drivers who were asked to drive instrumented vehicles for 7-27 days. Mefford et al. reported that $21 \%$ of the miles driven took place at night and during that time high beams were use only $3 \%$ of the time. Furthermore, even under ideal conditions (rural roads; no opposing or leading vehicle) drivers' high beam usage did not exceed $25 \%$. The findings reported by Buonarosa et al. confirmed that drivers' use of low beams far exceeds that of high beams $(97.6 \mathrm{~min} / 100 \mathrm{~km}$ vs. $9.8 \mathrm{~min} / 100 \mathrm{~km}$, respectively).

The present study is part of a larger project designed to assess young adults' understanding of the visual challenges drivers face at night and to improve high beam usage of these drivers. College students reported their high beam use over the course of several weeks; high beam use during an open-road drive was also recorded for these individuals. A low cost video-based method of gathering on-road high beam usage data was developed for the purposes of this study. Finally, the illuminance levels of each participant's low and high beam headlamps were 
measured in order to examine the relationship between beam output and beam usage. We hypothesized that participants who drove vehicles with relatively modest low beam output would tend to use their high beams more often.

\section{METHOD}

\section{Participants}

Forty-six Clemson University undergraduate students $18-25$ years of age $(M=19.9, S D=2.0)$ participated in this study. All participants possessed a valid driver's license and had access to a registered, insured vehicle. Additionally, all participants achieved a minimum binocular acuity of 6/12 (20/40) and reported having no known visual pathology (other than corrected refractive error).

\section{Procedure}

All participants were recruited to participate in a two-part study. During the first experimental session, participants completed a brief questionnaire and were informed that they would receive an emailed questionnaire daily to report various driving behaviors (e.g., use of navigational aid(s), listening to the radio), including high beam usage. Specifically, the questionnaire asked "If you drove at night, did you use your high beam headlights at any point?" Participants completed the daily questionnaire for the weeks that elapsed between the first and second experimental sessions $(M=23.1$ days $)$.

After at least three weeks elapsed, participants returned for the second experimental session that measured on-road high beam usage. Data were collected at least one hour after sunset and only on nights free of precipitation and fog. Participants drove their own vehicles to campus, where they were joined by two experimenters. They were told they would take a short drive around campus during which time a video camera would record what happened as they drove; they were not alerted to the fact that high beam usage was being monitored.

Two experimenters were always present in the participant's vehicle during data collection, with one riding in the front passenger seat and one in the rear seat. The experimenter in the front seat documented the participant's high beam usage throughout the drive, recording whether any leading or opposing vehicles were present on the road whenever high beams were activated. To measure high beam usage, the rear seat experimenter held and aimed a small video camera that recorded the dashboard and the forward view of the roadway as the participant drove a prespecified experimental route. The camera was connected to a laptop that recorded the video.

Prior to the drive, the rear seat experimenter briefly explained the procedure and answered any questions. Participants then drove the predetermined route that included a stretch designed to minimize both ambient illumination and traffic, therefore providing participants with several opportunities to use their high beam headlamps. This portion of the route was $2.6 \mathrm{~km}$ (1.6 miles) long and took participants approximately five minutes to complete (mean speed approximately $30 \mathrm{mph}$ ). After the drive, the illuminance (in lux) of the low and high beams of each participants' vehicle were measured (in an unilluminated parking lot) using a Minolta T-1 illuminance meter. 
Measurements were taken $30.5 \mathrm{~m}(100 \mathrm{ft})$ from the front of the midline of the vehicle at $15.2 \mathrm{~cm}$ above ground level.

Data from the video feed was later coded and compared to the other front seat experimenter's documentation of beam usage to ensure accurate calculation of the percentage of time high beams were used appropriately. An experimenter (not present during data collection trials) coded each video twice. During the first viewing, the experimenter recorded the total amount of time during the drive that high beam use would be recommended due to the absence of leading and oncoming vehicles. In the second viewing of each video, the amount of time each driver used their high beams was documented; instances of high beam use were determined by monitoring both the dashboard indicator and the beam pattern on the roadway.

\section{RESULTS}

On the daily questionnaire, participants self-reported an average of 2.6 (range: 0 to 11) instances of high beam usage over the course of three to four weeks. Out of the average 3 minutes and 8 seconds (range: $1 \mathrm{~min} 49 \mathrm{sec}-3 \mathrm{~min} 41 \mathrm{sec}$ ) during which high beam use was coded as "possible," participants used their high beams an average of 1 minute and 29 seconds (range: 0 min $0 \mathrm{sec}-4 \mathrm{~min} 10 \mathrm{sec}$ ). Thus, high beams were used $48 \%$ of the time that high beam use was possible. Note that one participant used their high beams more than was possible by our definition; this participant activated their high beams for the full duration of the drive regardless of the presence of other traffic.

The average illuminance level of participants' low beam headlights was 18.00 lux and 43.40 lux for high beam headlights. A one-tailed Pearson's correlation coefficient was calculated to investigate the potential inverse relationship between low beam illumination and high beam usage. The results of this analysis indicated that the relationship between low beam illumination and high beam usage was a moderately negative one, $r(44)=-.23, p=.06$ (see Figure 1 ), such that drivers whose low beams produced less illumination tended to use their high beams more often. Further, exploratory analyses revealed that there were no significant correlations between high beam illumination and high beam usage $(p>.05)$ nor between the difference in illumination between low and high beams and high beam usage $(p>.05)$.

\section{DISCUSSION}

The purpose of the present study was to document the high beam usage of young drivers. Drivers in this study used their high beams more often (on average) than drivers in previous studies. Both Hare and Hemion (1968) and Mefford, Flannagan, and Bogard (2006) reported that high beam usage did not exceed $25 \%$ even in ideal high beams conditions (e.g., rural, unlit roads with no traffic). In the present study, drivers used their high beams $48 \%$, on average, when no leading or oncoming vehicles were present. The route driven included several luminaires and high beam usage in this study still exceeded the rates reported by both Hare and Hemion and Mefford et al. These results reported here are, however, consistent with Sullivan et al. (2004), who reported that approximately half of drivers used their high beams when it was deemed appropriate. 


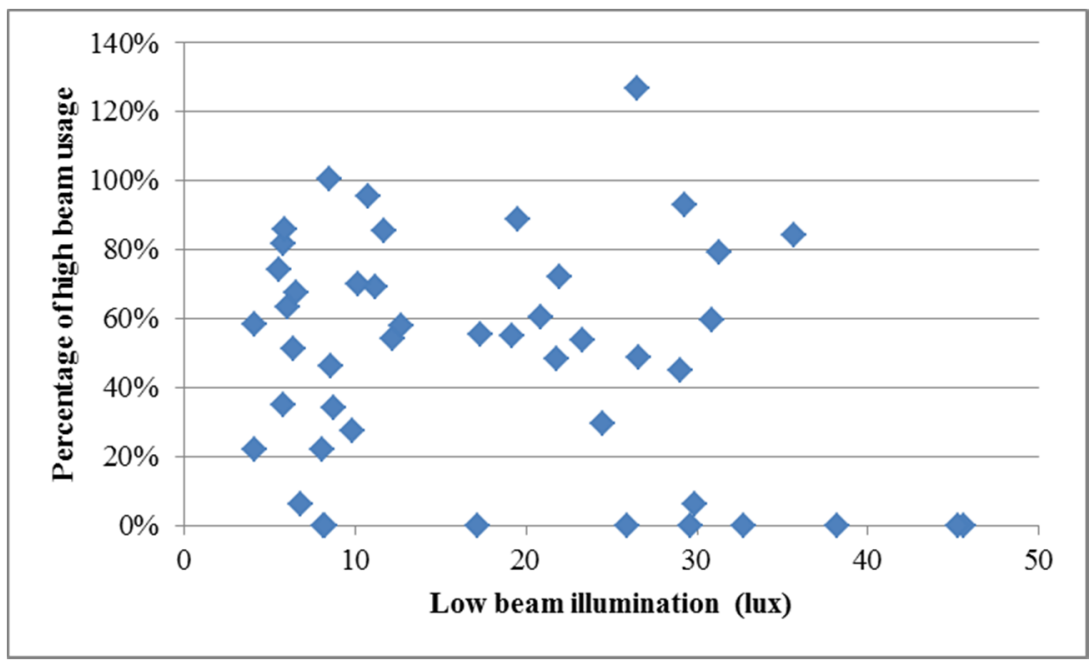

Figure 1. The relationship between low beam illumination (in lux) and on-road high beam usage across both groups

The modest correlation between low beam illumination and percentage of appropriate high beam usage provides further insight into the higher reported rates of high beam use in this study. There was a tendency for drivers whose low beams produced lower levels of illumination to use their high beams more often $(r=-.23)$. It is possible that the drivers of vehicles with decreased low beam output use their high beams more often to compensate for the relatively poor illumination provided by their low beams. An empirical investigation of the effect of decreased low beam illuminance on high beam usage would lend further insight into this relationship, particularly given that previous research suggests that drivers do not notice reductions in headlight illuminance until that reduction exceeds $60 \%$ (Rumar, 1974). However, participants in the Rumar study were simply asked about how dirty they thought their headlights were and if they intended to clean them; no measures of driver behavior were recorded. It is possible that smaller reductions in headlight illuminance (i.e., less than $60 \%$ ) may be sufficient to change driver behaviors such as high beam usage. Relatedly, the effect of headlamp type (high intensity discharge (HID) vs. halogen) on high beam usage should be examined given evidence (e.g., Sivak, Flannagan, Schoettle, \& Nakata, 2002) that HID headlamps produce more illumination in a wider beam pattern than halogen headlamps. Drivers using HID low beams may be less inclined to use their high beams due to this difference in illuminance and beam distribution.

Though drivers in this study used their high beams relatively often, there are limitations associated with this study. While the drivers were not informed of the purpose of the in-vehicle camera prior to the drive, the fact that their driving was being monitored may have encouraged them to engage in "good behavior." Because the participants were asked to fill out a daily survey about driving behaviors, which included questions about high beam usage, they may have been alerted to the fact that using their high beams would be considered a "good behavior" in this study. During the drive in the second session, two participants (4\% of sample) mentioned that they thought their high beam use was being monitored since the daily questionnaire had asked about this behavior. This suggests that other participants may also have been aware of the true purpose of the study, artificially inflating high beam usage rates. Similarly, Lavin and Groarke (2005) reported that diary keeping may create demand characteristics, thereby influencing 
participants to behave differently than they might otherwise. Additionally, the test route that participants drove was relatively short and data gathered may not be predictive of drivers' typical high beam usage when traveling longer distances.

Despite these limitations, the present study offers two key benefits in respect to measuring onroad high beam usage. First, these data provide driving researchers with updated data regarding drivers' high beam use during an open-road drive. Here drivers used their high beams more often than previously reported and further research is needed to confirm the accuracy of this finding. The second benefit of the current research is the method employed to measure high beam usage. This method had high external validity, in that drivers drove an open-road route that was carefully chosen based on several key factors thought to influence high beam usage (e.g., roadway with low traffic volume and a general absence of roadway illumination). This approach allowed for greater control of such factors while also gathering naturalistic driving data. This methodology may be applied to future research investigating not only high beam usage but a variety of driving behaviors (e.g., distracted driving) that can be recorded via video feed from the driver's perspective.

Future research should continue to investigate the factors that influence drivers' use of high beams and ways in which an appropriate reliance on high beams can be developed. This area of research will be particularly relevant as more vehicle manufacturers begin to incorporate adaptive headlights (i.e., headlights that change aim and/or modify their output in accordance with traffic conditions as drivers navigate roadways; Fleming, 2012) into vehicles.

Understanding drivers' knowledge of the benefits of high beams may influence both their trust and use of these developing technologies. For example, drivers who believe that low beams are sufficient for all nighttime conditions may choose to disable an adaptive system that adds light where they believe that light should not be projected. Further research is also needed to understand how educating drivers about the challenges associated with driving at night may impact the use of technology such as adaptive headlights and how headlight use relates to driving safely at night.

\section{ACKNOWLEDGMENTS}

The first author is now a Human Factors Consultant with Applied Building Sciences, Inc. The authors are grateful for the assistance of Stanton Adams, Larry Donaldson, Drea Fekety, Xinyu Liu, and Keanau Ormson throughout the course of data collection.

\section{REFERENCES}

Allen, M.J., Hazlett, R.D., Tacker, H.L, \& Graham, B.V. (1969). Actual pedestrian visibility and the pedestrian's estimate of his own visibility. American Journal of Optometry and Archives of American Academy of Optometry, 44-49.

Balk, S.A., Tyrrell, R.A., Brooks, J.O., \& Carpenter, T.L. (2008). Highlighting human form and motion information enhances the conspicuity of pedestrians at night. Perception, 37, 12761284.

Buonarosa, M.L., Sayer, J.R., \& Flannagan, M.J. (2008). Real-world frequency of use of automotive lighting equipment. Leukos, 5, 139-146. 
Fleming, B. (2012). New automotive electronics technologies. IEEE Vehicular Technology Magazine, 7(4), 4-12.

Hare, C.T. \& Hemion, R.H. (1968). Headlamp beam usage on U.S. highways. Final report on phase III (AR-66). San Antonio, TX: Southwest Research Institute.

Helmers, G. \& Rumar, K. (1975). High beam intensity and obstacle visibility. Lighting Research and Technology, 7(1), 35-42.

Lavin, D. \& Groarke, A. (2005). Dental floss behaviour: A test of the predictive utility of the Theory of Planned Behaviour and the effects of making implementation intentions. Psychology, Health, \& Medicine, 10(3), 243-252.

Leibowitz, H., Owens, D., \& Tyrrell, R. A. (1998). The assured clear distance ahead rule: Implications for nighttime traffic safety and the law. Accident Analysis and Prevention, 30(1), 93-99.

Mefford, M.L., Flannagan, M.J., \& Bogard, S.E. (2006). Real-world use of high-beam headlamps (No. UMTRI-2006-11). Ann Arbor, MI: The University of Michigan Transportation Institute.

Olson, P.L. \& Sivak, M. (1983). Comparison of headlamp visibility distance and stopping distance. Perceptual and Motor Skills, 57, 1177-1178.

Owens, D.A. \& Sivak, M. (1996). Differentiation of visibility and alcohol as contributors to twilight road fatalities. Human Factors, 38(4), 680-689.

Roper, V.J. \& Howard, E.A. (1937). Seeing with motorcar headlamps. Presented at the $31^{\text {st }}$ Annual Convention of the Illuminating Engineering Society, White Sulphur Springs, WV.

Rumar, K. (1974). Dirty headlights - frequency and visibility effects. Ergonomics, 17(4), 529533.

Rumar, K. (2000). Relative merits of the U.S. and ECE high-beam maximum intensities and of two- and four-headlamp systems (No. UMTRI-2000-41). Ann Arbor, MI: The University of Michigan Transportation Research Institute.

Sivak, M. Flannagan, M.J., Schoettle, B., \& Nakata, Y. (2002). Performance of the first generation of HID headlamps in the U.S. (No. UMTRI-2002-14). Ann Arbor, MI: The University of Michigan Transportation Research Institute.

Sullivan, J.M., Adachi, G., Mefford, M.L., \& Flannagan, M.J. (2004). High-beam headlamp usage on unlighted rural roadways. Lighting Resources Technology, 36, 59-67.

Sullivan, J.M. \& Flannagan, M.J. (2001). Characteristics of pedestrian risk in darkness (No. UMTRI-2001-33). Ann Arbor, MI: The University of Michigan Transportation Research Institute.

Sullivan, J.M. \& Flannagan, M.J. (2002). The role of ambient light level in fatal crashes: Inferences from daylight saving time transitions. Accident Analysis and Prevention, 34(4), 487-498.

Wood, J.M., Tyrrell, R.A., \& Carberry, T.P. (2005). Limitations in drivers' ability to recognize pedestrians at night. Human Factors, 47(3), 644-653. 